\title{
Research on PID Controller in Active Magnetic Levitation Based on Particle Swarm Optimization Algorithm
}

\author{
Zhang Yanhong ${ }^{1,2, *}$, Zheng Zhongqiao ${ }^{1,3}$, Zhang Jiansheng ${ }^{1}$ and Yin Lei ${ }^{1}$
}

${ }^{1}$ School of Electrical and Photoelectronic Engineering, Changzhou Institute of Technology, Changzhou, Jiangsu, 213002, China; ${ }^{2}$ School of Electrical and Information Engineering, Jiangsu University, Zhenjiang, Jiangsu, 212013, China; ${ }^{3}$ School of Mechatronic Engineering and Automation, Shanghai University, BaoShan District, Shanghai, 200444, China

\begin{abstract}
Active magnetic levitation control system is an open-loop and unstable control system. The parameters of PID controller are usually set according to the experience of the designer. It is not only time-consuming and tedious, but also can't be controlled accurately. So the particle swarm optimization algorithm is used in this paper, which is used to optimize the parameters of PID controller by combining with PID controller. The proportional coefficient, integral coefficient and differential coefficient of PID controller are used as three particles of the particle swarm optimization algorithm. The accurate initialization parameters are selected, and then the speed and position information is updated to realize the global optimization of particle. Therefore, the three optimized parameters of PID controller are obtained, which are used to simulate the active magnetic levitation control system. The simulation result shows that the optimized parameters can make the active magnetic levitation control system stable.
\end{abstract}

Keywords: Active magnetic levitation, Global optimization, Particle swarm optimization algorithm, PID controller, Stability.

\section{INTRODUCTION}

Active magnetic levitation control system has some advantages, such as no contract, no friction, long life and controllable supporting force. So it has been widely used in the industry. The core is controller of the active magnetic levitation control system. The structure of PID controller is simple, the design method is very mature and it has better control effect for the linear control system. Therefore, PID controller is widely used in industrial control system. PID controller is also used in the active magnetic levitation control system, but the adjustment of the proportion coefficient, integral coefficient and differential coefficient have always been a problem. If the parameter is not appropriate, the active magnetic levitation control system will be unstable. Therefore, how to select some optimization algorithms to make the three parameters of PID controller adjust online is a hot issue in recent years. A kind of improved PID controller in the active magnetic levitation has been studied in document [1]. The control performance of the system has been improved. A kind of fuzzy and adaptive PID controller has been studied in document [2], and the parameters of PID controller are set by establishing fuzzy rules. A kind of PID controller based on the neural network has been studied in document [3], in which the parameters of PID controller are set by neural network. Some better results have been gained by different methods in several documents. The particle swarm optimization algorithm has been introduced and the three

*Address correspondence to this author at the School of Electrical and Photoelectronic Engineering, Changzhou Institute of Technology, Changzhou, Jiangsu, 213002, China; Tel: +8651985217623;

Fax:+8651985217620; E-mail: zhangyhczu@163.com parameters of PID controller are used as three particles. The three optimized parameters of PID controller are obtained by updating the position and speed of the particles. The simulation model of the active magnetic levitation control system has been established by Simulink. The three optimized parameters of PID controller are transferred to the simulation model, then the established system is simulated, which realizes the stable control of the active magnetic levitation system.

\section{THE COMPOSITION OF THE ACTIVE MAGNET- IC BEARING}

The structure of the active magnetic bearing system is mainly composed of the current displacement sensor, the rotor, the axial magnetic bearing, the radial magnetic bearing, the thrust plate, the driving motor and the protection bearing. The structure is shown in Fig. (1).

The active magnetic bearing system has five degrees of freedom. They are respectively two horizontal degrees of freedom, two vertical degrees of freedom and one axial degree of freedom. The axial magnetic bearing is installed in the middle of thrust plate, which makes the system balance in the axial direction. The two radial magnetic bearings are respectively installed at the left and the right end of the rotor, and they make the rotor balance in the horizontal and vertical directions. The two protection bearings are located at both ends of the rotor. When the magnetic bearing system does not work, the rotating shaft should be placed on the protecting bearing, which reduces the air gap between the protection bearing and the rotor. When the active magnetic levitation bearing system is out of control, the protection bearing supports the high-speed rotating rotor and prevents 


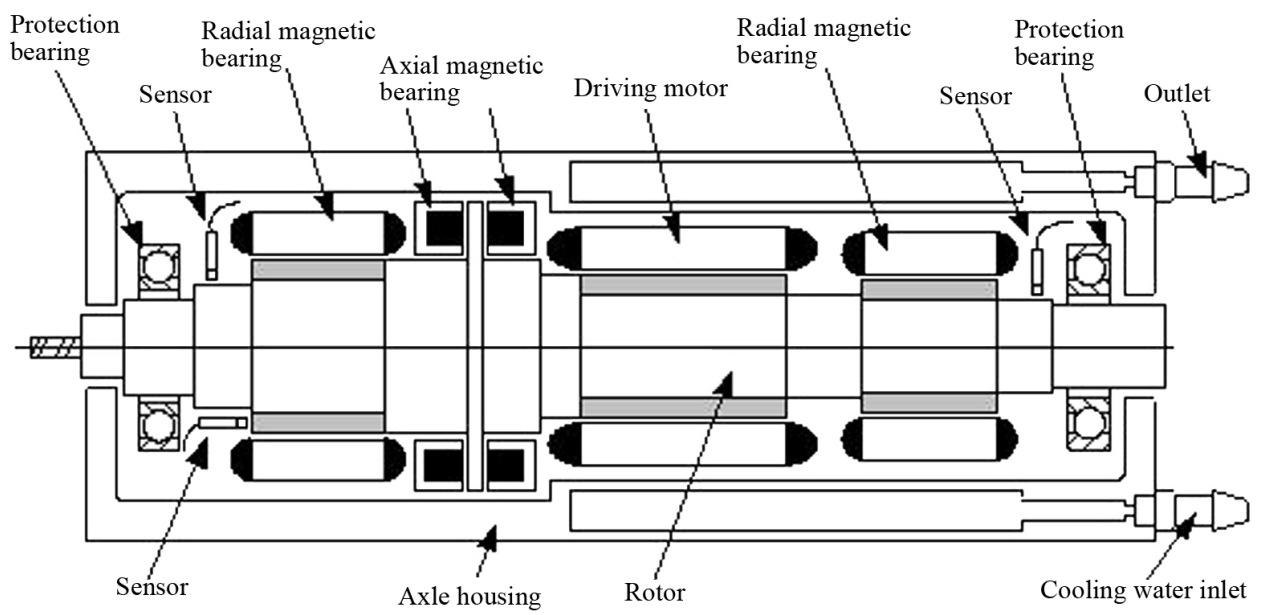

Fig. (1). Structure of the active levitation bearing.

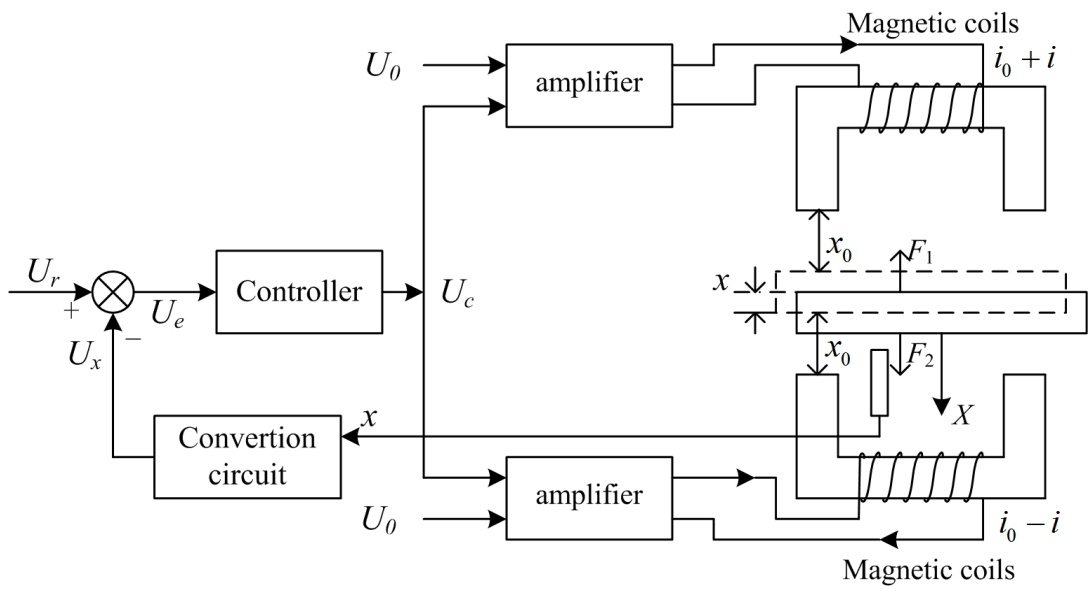

Fig. (2). Structure of the active magnetic levitation system with a degree of freedom.

damaging the whole magnetic levitation bearing system because of the collision of the rotor and the stator [4-6].

\section{MATHMATIC MODEL OF ACTIVE MAGNETIC LEVITATION WITH A DEGREE OF FREEDOM}

The structure of the active magnetic bearing system with a degree of freedom is shown as Fig. (2).

The quality of the rotor is $\mathrm{m}$. The rotor locates between upper electromagnet and lower electromagnet, and only the bearing force in the vertical direction is considered. When the rotor is in equilibrium, the following assumption is made.

(1) The winding leakage magnetic flux is ignored.

(2) The reluctance of the iron core and the rotor are ignored, namely the magnetic potential is only in the air gap.

(3) The hysteresis and the eddy current effect of the magnetic material is ignored.

Suppose that a disturbance appears, which makes the rotor deviate the equilibrium position $\mathrm{x}$. In order to make the rotor return to its equilibrium position, a control current is produced by the electromagnet, which reduces the electromagnetic force of the upper electromagnet and increases the electromagnetic force of the lower electromagnet, and the electromagnetic force is expressed as follows.

$$
F=F_{1}-F_{2}=\frac{\mu_{0} S N^{2}}{4}\left[\frac{\left(i_{0}-i_{c}\right)^{2}}{\left(x_{0}-x\right)^{2}}-\frac{\left(i_{0}+i_{c}\right)^{2}}{\left(x_{0}+x\right)^{2}}\right]
$$

Where, $\mu_{0}$ is the magnetic permeability of vacuum, which unit is $\mathrm{H} / \mathrm{m} . S$ is the cross-sectional area of the magnet, which unit is $\mathrm{mm}^{2} . \mathrm{N}$ is the number of turns of electromagnetic coil, $i_{0}$ is the bias current of the electromagnetic coil, which unit is A. $x_{0}$ is the air gap between the rotor and the magnet when the rotor is in equilibrium, which unit is $\mathrm{mm}$.

According to Newton's second law, the force of the rotor is expressed as follows.

$$
F=m a=m \frac{d^{2} x}{d t^{2}}
$$

Where, $F$ is the electromagnetic force, which is the function of the displacement and the current, $\mathrm{m}$ is the quality of the rotor and $x$ is the displacement.

The formula (2) is substituted into the formula (1) and the following formula can be obtained. 


$$
\frac{\mu_{0} S N^{2}}{4}\left[\frac{\left(i_{0}-i_{c}\right)^{2}}{\left(x_{0}-x\right)^{2}}-\frac{\left(i_{0}+i_{c}\right)^{2}}{\left(x_{0}+x\right)^{2}}\right]=m \frac{d^{2} x}{d t^{2}}
$$

It can be seen from formula (3) that the mathematical model of the magnetic bearing is a second order nonlinear differential equation. It is difficult to calculate the solution of formula (3). In the practical application, the nonlinear control system is often solved by transforming into the linear control system. Therefore, the electromagnetic force is linearized by Taylor formula in the neighborhood of the equilibrium point, the following formula is obtained.

$$
\begin{aligned}
& F\left(i_{c}, x\right) \approx-k_{i} \cdot i_{c}+k_{x} \cdot x \\
& k_{i}=\frac{\mu_{0} S N^{2} i_{0}}{x_{0}^{2}}, k_{x}=\frac{\mu_{0} S N^{2} i_{0}^{2}}{x_{0}^{3}}
\end{aligned}
$$

Where, $k_{i}$ is the current coefficient, $k_{x}$ is the displacement coefficient.

It can be seen from formula (5), when the structural parameters of the system and the static working point $\left(i_{0}, x_{0}\right)$ are determined, where $k_{i}$ and $k_{x}$ are both constants.

It can be obtained from formulas (2) and (4)

$$
-k_{i} \cdot i_{c}+k_{x} \cdot x=m \ddot{x}
$$

The Laplace transform is made in the formula (6), the transfer function can be obtained as follows.

$$
G(s)=\frac{X(s)}{I(s)}=\frac{-k_{i}}{m s^{2}-k_{x}}
$$

It can be seen from formula (7) that a characteristic root is located in the right half plane, and the system is unstable, so the controller must be designed.

\section{PARTICLE SWARM OPTIMIZATION ALGO- RITHM}

Particle swarm optimization algorithm (PSO) was proposed by Jim Kennedy and Eberhart in 1995. It is derived from the predatory behavior of birds. A group of birds search for food in the random, if there is only a piece of food, the most simple and effective strategy of finding the food is to search for the surrounding area of birds who are nearest to food. PSO algorithm is obtained from this model which is used to solve the optimization problem [6-8]. In the particle swarm algorithm, each individual is called as one particle, and each particle represents a potential solution. All particles have a fitness value determined by the optimization function, with each particle having a speed, which determines the direction and distance of the particle. Other particles follow the optimal particle to search for the extreme value in the solution space. According to the set fitness function in advance, the current fitness value is calculated. The position of the particle is weighed by whether the fitness value is optimal. In each iteration, the particles are updated by tracking two extreme values. One is the optimal solution, which is called as the individual extreme value, namely pBest; the other is the best solution of the whole particle swarm currently, which is called as the global extreme value, namely gBest. The initialization of PSO is a group of random particle, which optimal solution is found by iteration. The neighbors of the part optimum particles can be used and the extreme of all neighbors is a local extreme value.

Because PSO algorithm is simple and easy to realize, it has been more and more applied in some fields, such as the function optimization, the neural network training, the pattern classification and the traditional optimization algorithm. The convergence speed of PSO algorithm is faster, but it is easy to fall into the local optimum, which is related to the selection of the inertia factor $\omega$. According to experience, the greater the inertia weight factor of the particle is, the better the global optimum of the particle is, since the smaller $\omega$ is better for the algorithm convergence. Therefore, at the beginning of optimization, it is hoped that the larger inertia factor makes the scope of optimization wide. At the last stage of optimization, the smaller inertia factor is hoped to make the convergence better. The Flow of PSO algorithm is shown as Fig. (3).

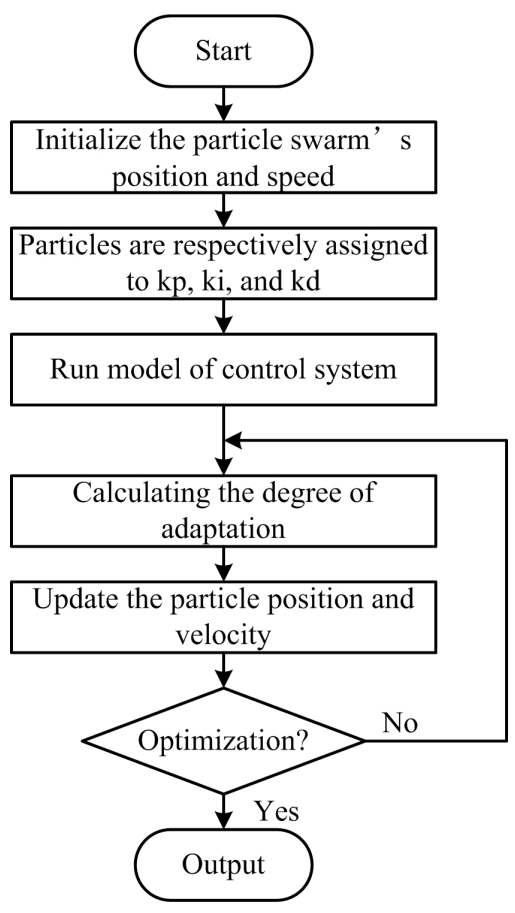

Fig. (3). Flow of PSO algorithm.

Suppose that there is a population of $\mathrm{m}$ particles, where the position of $i$ particle is $p_{i}=\left(p_{i 1}, p_{i 2}, \cdots p_{i m}\right)$, the velocity is $v_{i}=\left(v_{i 1}, v_{i 2}, \cdots v_{i m}\right)$, the updating formula of position and velocity is as follows.

$$
\begin{aligned}
& V_{i}^{k+1}=\omega V_{i}^{k}+c_{1} r_{1}\left(P_{A}-p_{i}^{k}\right)+c_{2} r_{2}\left(P_{B}-p_{i}^{k}\right) \\
& p_{i}^{k+1}=p_{i}^{k}+v_{i}^{k}
\end{aligned}
$$

Where, $i=1,2, \cdots, m, m$ is the scale of the particle swarm. $k$ is the iterative number of algorithm, $V_{i}^{k}$ represents the speed of i particle when the iteration number is $k, p_{i}^{k}$ represents the position of $i$ particle when the iteration number is $k$, $r_{1}$ and $r_{2}$ are the random numbers distributed in the range of $[0,1], c_{1}$ and $c_{2}$ are the nonnegative constants, which are called as the acceleration factor. $\omega$ is the inertia factor. $P_{A}$ indicates the optimal position of a particle in the particle group, $P_{B}$ is the optimal position of the particle group. 


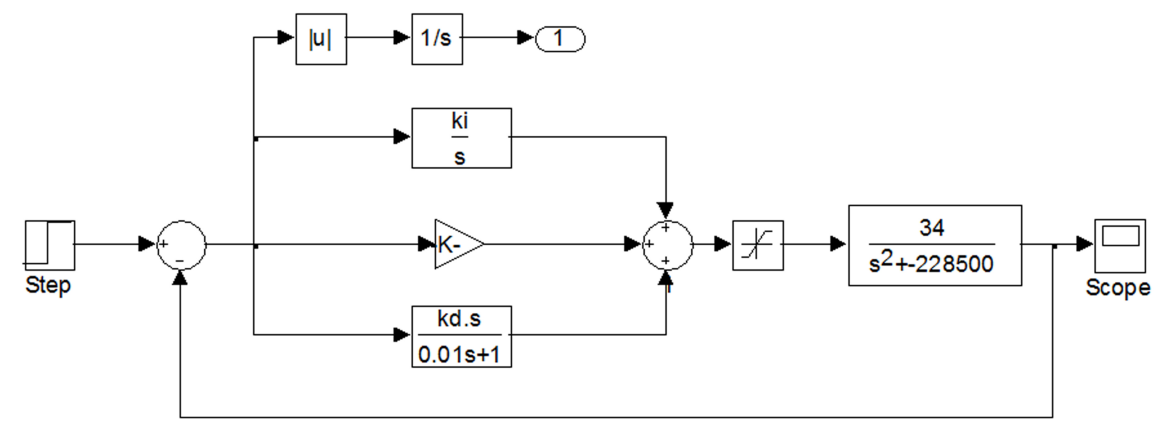

Fig. (4). Simulation model of PID control system.

\section{THE SIMULATION AND ANALYSIS}

In the active magnetic levitation system, the mass of the rotor is $\mathrm{m}=10 \mathrm{~kg}$. The equilibrium position of the rotor is $x_{0}$ $=0.3 \times 10^{-3} \mathrm{~m}$. The bias current of the electromagnetic coil is $I_{0}=2.0 \mathrm{~A}$. The magnetic permeability of the air gap is $\mu_{0}=$ $4 \pi \times 10^{-7} \mathrm{Vs} / \mathrm{Am}$. The section area of the magnetic pole is $s_{0}=$ $340 \mathrm{~mm}^{2}$. The number of turns of the electromagnetic coil is $\mathrm{N}=190$. These parameters are substituted into the equation (7), so that the open-loop transfer function of the active magnetic levitation control system can be obtained as follows.

$$
G(s)=-\frac{34}{s^{2}-22.85 \times 10^{4}}
$$

The PID controller of incomplete differential is adopted. The performance index is $J=\int_{0}^{\infty}|e(t)| d t$. The initial parameters of PSO are set. The size of the particle swarm is 100 . The acceleration constant is respectively $c_{1}=1.5, c_{2}=2$. The range of the velocity is $[-1,1]$. The number of optimized parameters is 3 , namely three parameters of $k_{p}, k_{i}$ and $k_{d}$. The range of the optimized parameters is $[0,100]$. The inertia weight coefficient is $\omega=0.8$, and the maximum of the iteration is 100. The PID controller based on PSO is realized by MATLAB and the system model is established by Simulink [9-11], which is shown as Fig. (4).

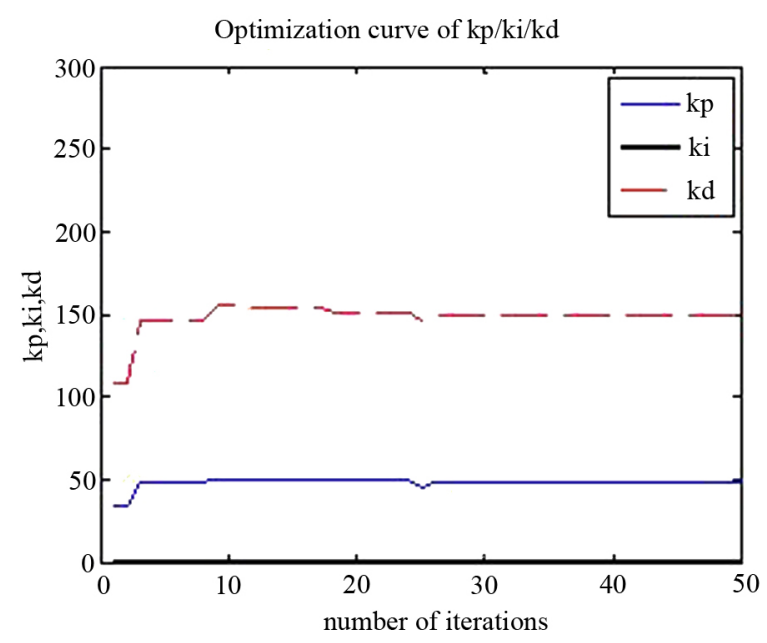

Fig. (5). The optimization curve of PID parameters.

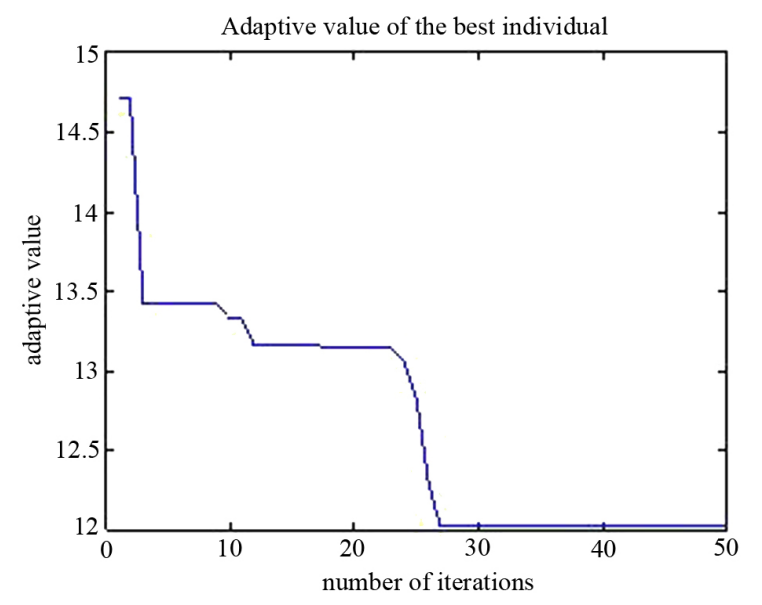

Fig. (6). The adaptive value of the best individual.

The values of three particles are passed to $k_{p}, k_{i}$ and $k_{d}$, the output value is observed from the oscilloscope and the performance index is output from the output terminal. The change curves of $k_{p}, k_{i}$ and $k_{d}$ are shown as Fig. (5). The best individual fitness value is shown in Fig. (6). When the parameters of PID controller are optimal, the output curve of the control system is shown as Fig. (7).

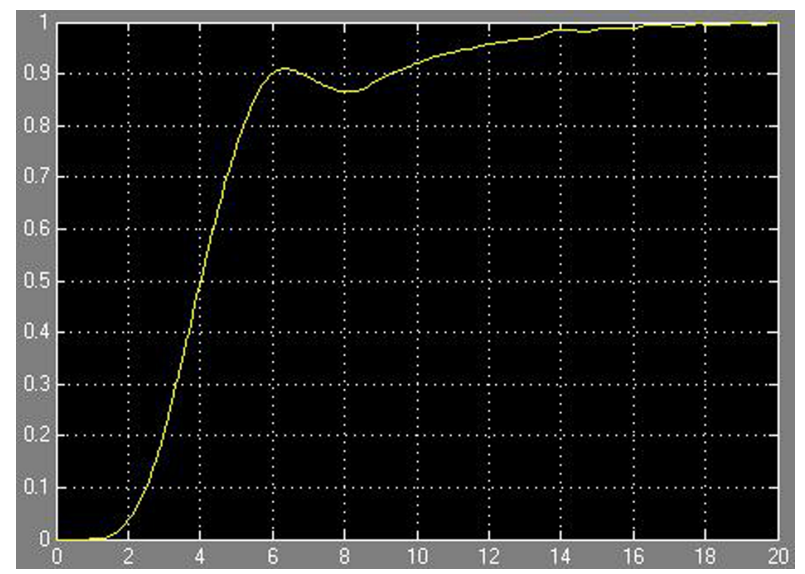

Fig. (7). The output curve of the control system.

It can be seen from (Fig. 5) that the optimal parameters of PID controller are $k_{\mathrm{p}}=48.85, k_{\mathrm{i}}=0.1746$ and $k_{\mathrm{d}}=149.36$ after 25 iterations. It is also seen from (Fig. 6) that the best individual fitness value continuously decreases at the beginning, the value tends to be 12.05 until the number of the iter- 
ation is 27. The parameters of PID controller are used in the simulation model. It can been seen from Fig. (7) that the output of the control system tends to be stable, which can be proved by that the PSO algorithm is effective in the active magnetic levitation control system.

\section{CONCLUSION}

Because the active magnetic levitation system has some characteristics, such as the uncertainty of parameters, nonlinearity etc. In order to solve the difficult problem of the parameters tuning in the traditional PID controller, in the active magnetic levitation control system, the particle swarm optimization algorithm is combined with the conventional PID controller. PID controller is designed based on particle swarm optimization algorithm. The proportional coefficient, integral coefficient and differential coefficient of PID controller are regarded as three particles. At the same time, the performance index of IAE is used to minimize the position error of the rotor. The parameters of PID controller are adjusted online. PID controller based on PSO can make the active magnetic levitation system more stable. It is found by the experiment that the fixed inertial coefficient of particle swarm algorithm has a great influence on the optimal range of particle. The larger the inertia coefficient $\omega$ is, the better the global optimal of particle is, and the smaller the inertia coefficient $\omega$ will be, which laid the foundation for subsequent research.

\section{CONFLICT OF INTEREST}

The authors confirm that this article content has no conflict of interest.

\section{ACKNOWLEDGEMENTS}

This work is supported by the National Natural Science Foundation of China (Grant No. 51175052 and No.51405040), the basic application and research program of Changzhou (Grant No. CJ20130014). The Natural Science Foundation of Jiangsu Province (Grant No. BK20151182).

\section{REFERENCES}

[1] X. Wang, and G. Zhang, "Magnetic levitation bearing control system simulation based on variable universe fuzzy PID algorithm," Instrument Technique and Sensor, vol. 12, pp. 144-147, 2012.

[2] J. Zhang, and L. Xu, "Fuzzy adaptive PID control of maglev system," Journal of Computer Applications, vol. 29, no. 6, pp. 329$334,2009$.

[3] J. Zhang, X. Pei, and H. Xing, "Improved RBF network control," Journal of Harbin University of Science and Technology, vol. 16, no. 1, pp. 48-52, 2011

[4] K. Shen, and C. Sun, "Research on an active magnetic suspension system with Matlab simulation," Industrial Instrumentation and Automation Devices, vol. 4, pp. 7-11, 2008.

[5] E. Shameli, M. B. Khamesee, and J. P. Issoon, "Nonlinear controller design for a magnetic levitation device," Microsystem Technologies, vol. 13, no. 5, pp. 831-835, 2007.

[6] M. Zhang, "Study on the application of the improved particle swarm intelligent algorithm and multi-objective optimization," [unpublished].

[7] Z. Lv, and Z. Hou, "Particle swarm optimization algorithm with adaptive mutation," Chinese Journal of Electronics, vol. 32, no. 3, pp. 416-420, 2004.

[8] L. Zhang, H. Yu, and D. Chen, "Analysis and improvement of particle swarm optimization algorithm," Information and Control, vol. 33, no. 5, pp. 513-517, 2004.

[9] F. Shi, H. Wang, and L. Guo, MATLAB Intelligent Algorithm, $1^{\text {st }}$ ed. Beihang University Press: Beijing, 2010.

[10] J. Liu, Advanced PID Control and Matlab Simulation, $1^{\text {st }}$ ed. Publishing House of Electronics Industry: Beijing, 2003.

[11] W. Luo, J. Han, and H. Lan, "Advanced simulation of fuzzy control Based on the Matlab (Simulink) language," Computer Simulation, vol. 18, no. 3, pp. 15-16, 2001.

(C) Yanhong et al.; Licensee Bentham Open.

This is an open access article licensed under the terms of the (https://creativecommons.org/licenses/by/4.0/legalcode), which permits unrestricted, noncommercial use, distribution and reproduction in any medium, provided the work is properly cited. 